An attempt has been made to correlate the yield points and elastic limits with microstructure. The relationship suggested by Gensammer and coworkers between flow stress, tensile strength or hardness of steels and the mean ferrite path prompted an initial investigation of this microstructure parameter. Mean ferrite paths have been determined for the series of steels and pure irons by a lineal analysis method employing a travellng microscope stage and a Hurlbut counter.

The results obtained to date show that:

1. Residual stress lowers the elastic limit, rounds off the yleld point phenomenon, and raises the yield point stress considerably.

2. No plastic relaxation effects are observed until a plastic strain of at least $5000 \times 10^{-1}$ has been applied and the magnitude of the relax- ation is then of the order 25 to $50 \times 10^{-6}$.

3. A step-like behavior is obtained in the initial plastic strain curves of materials which contain no free ferrite.

4. For stress-relieved materials the yield point is a linear function of the logarithm of the mean ferrite path. This relation holds over the range of the logarithm equal to 3.8 to 5.5 , where ferrite paths are in angstrom units. Grain boundaries, carbide particles, pearlite patches and inclusions are considered terminations of the ferrite paths.

5. A similar relation has been found for the elastic limit of stress-relieved materials but the linearity does not appear to hold well in the end of the range corresponding to higher strength and shorter mean ferrite path.

\title{
On the $\{259\}_{\text {y }}$ Habit of Martensite
}

\author{
by Eugene S. Machlin
}

$\mathbf{M}$ EASUREMENTS were made of the non-diagonal components of the martensite transformation strain matrix using single crystals of a 70 pct $\mathrm{Fe}, 30$ pct $\mathrm{NI}$ alloy. The habit planes associated with each strain matrix were determined simultaneously, and were found to belong closely to the $\{259\} \gamma$ family. A matrix method of analysis yielded the following conclusions:

1. The hablt plane is the only plane of no distortion and no rotation associated with the measurable strain of the martensite plate.

2. To each measured strain matrix there corresponds one and only one permutation of \pm $\{259\}_{\gamma}$ and vice versa.

3. There are 24 measurably different strain matrices. These matrices can be arranged in two groups (12 in each group). These groups have the property that any strain matrix belonging to one group is crystallographically equivalent to every other strain matrix belonging to that group. One group is related to the other by the property that any strain matrix belonging to one group has a crystallographic mirror symmetry with every strain matrix belonging to the other group.

4. The reaction path (motion of the atoms during the transformation) is the same crystallographically for every martensite plate formed.

5. There are at least two homogeneous strains required to form the martensitic structure. The first comprises the measurable strain. The second is homogeneous only over a small region and is not measurable. The elements of these strains are:

\begin{tabular}{|c|c|c|}
\hline $\begin{array}{l}\text { Plane of zero distortion } \\
\text { and zero rotation }\end{array}$ & $\begin{array}{l}\text { Ist Strain } \\
(259) \gamma\end{array}$ & $\begin{array}{l}\text { 2nd Strain* } \\
\text { Between }(112) \text { s and }(011) \text { s } \\
\text { and } 61 / 2 \circ \text { from (112) sx. }\end{array}$ \\
\hline $\begin{array}{l}\text { Direction of motion of plane } \\
\text { of zero distortion and zero } \\
\text { rotation }\end{array}$ & $\begin{array}{l}\text { Between }\left[0 \Gamma^{\top 1}\right] \gamma \\
\text { and }[112] \gamma \text { and } \\
\sim 10^{\circ} \text { from }\left[0 I^{\top} 1\right] y\end{array}$ & $\begin{array}{c}\sim 2 \frac{1}{2} \text { from }[1 \mid \bar{T}] \mathbf{M}, \\
\text { coincident } \\
\text { with }[1 \overline{1} 0] \gamma .\end{array}$ \\
\hline
\end{tabular}

* This strain is based on the assumption of a Greninger-Troiano orientation relation between the parent austenite and the martensite.

6. There are four martensite habit planes having directions of motion of the bounding austenite within $10^{\circ}$ of any $<110>\gamma$. This fact is used to account for the burst effect in $\{259\} \gamma$ systems.

7. The difference between the elements of motion yielding $\{225\}_{\gamma}$ habits and those yielding $\{259\}_{\gamma}$ habits is the presence of shear components yielding rotations of directions about [111] $]_{\gamma}$ in the latter system. A correlation is suggested as follows: (225) $\gamma$ Kurdjumow-Sachs orientation

(259) $y$ Greninger-Trolano orientation

8. The angles between martensite plates in the $\{225\}_{\gamma}$ and $\{259\}_{\gamma}$ systems are dependent upon the bounding plates having the same direction of austenite motion.

9. Finally, the sequence of events determining the choice of habit plane is believed to be as follows: During the martensite transformation the atoms move with respect to each other along a unique reaction path of minimum potential energy. During the first homogeneous strain, each atom moves along a straight line path. At any point along this reaction path, the strain matrix has only one plane of no distortion and no rotation associated with it. It is the same plane at any point along the reaction path. Because the surface and coherency energy associated with this plane as an interface between austenite and martensite is the least of all possible planes, the strain spreads along this plane into the austenite to form the martensite plate. This plane of zero distortion and zero rotation is then the interface plane and also the habit plane.

E. S. Machlin is associated with Massachusetts Institute of Technology, Cambridge, Mass. 